\title{
Complement factor $D$ is linked to platelet activation in human and rodent sepsis
}

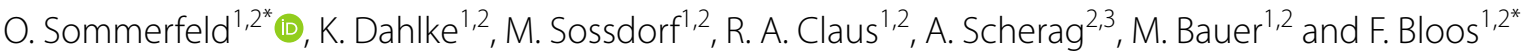

\section{*Correspondence:}

Oliver.Sommerfeld@med.unijena.de; Frank.Bloos@med.

uni-jena.de

${ }^{1}$ Department

of Anesthesiology

and Intensive Care Medicine,

Jena University Hospital,

Jena, Germany

Full list of author information

is available at the end of the article

\begin{abstract}
Background: The complement factor D (CFD) exerts a regulatory role during infection. However, its physiological function in coagulopathy and its impact on the course of an infection remains unclear.
\end{abstract}

Materials: Wild-type and CFD-deficient mice $(n=91)$ were subjected to cecal ligation and puncture to induce sepsis. At several time points, markers of coagulation and the host-immune response were determined. Furthermore, in patients $(n=79)$ with sepsis or SIRS, CFD levels were related to clinical characteristics, use of antiplatelet drugs and outcome.

Results: Septic CFD-deficient mice displayed higher TAT complexes ( $p=0.02)$, impaired maximal clot firmness, but no relevant platelet drop and reduced GPIIb/ Illa surface expression on platelets $(p=0.03)$ compared to septic wild-type mice. In humans, higher CFD levels (non-survivors, $5.0 \mu \mathrm{g} / \mathrm{ml}$ to survivors, $3.6 \mu \mathrm{g} / \mathrm{ml} ; p=0.015$ ) were associated with organ failure (SOFA score: $r=0.33 ; p=0.003)$ and mortality $(75 \%$ percentile, $61.1 \%$ to $25 \%$ percentile, $26.3 \%$ ). CFD level was lower in patients with antiplatelet drugs $(4.5-5.3 \mu \mathrm{g} / \mathrm{ml})$ than in patients without.

Conclusion: In mice, CFD is linked to pronounced platelet activation, depicted by higher GPIIb/IIla surface expression in wild-type mice. This might be of clinical importance since high CFD plasma concentrations were also associated with increased mortality in sepsis patients.

Keywords: Alternative complement pathway, Complement factor D, Coagulopathy, Infection

\section{Introduction}

During infection, invading microorganisms activate the host response where the complement system is part of the "first line of defense". Complement activation occurs via the classical, alternative or lectin pathways. A deficiency in one of the three pathways is associated with increased mortality, impaired bacterial clearance and high cytokine levels [1]. Several of such conditions are known: (1) genetic deficiencies of the classical pathway are associated with autoimmune diseases. Such diseases are accompanied with impaired humoral response as well as ineffective elimination of immune complexes, apoptotic materials, and necrotic debris [2]. (2) Deficiencies of components of the alternative (complement factor $\mathrm{D}$, properdin) and of the lectin (MBL) pathways impair

(c) The Author(s), 2021. Open Access This article is licensed under a Creative Commons Attribution 4.0 International License, which permits use, sharing, adaptation, distribution and reproduction in any medium or format, as long as you give appropriate credit to the original author(s) and the source, provide a link to the Creative Commons licence, and indicate if changes were made. The images or other third party material in this article are included in the article's Creative Commons licence, unless indicated otherwise in a credit line to the material. If material is not included in the article's Creative Commons licence and your intended use is not permitted by statutory regulation or exceeds the permitted use, you will need to obtain permission directly from the copyright holder. To view a copy of this licence, visit http:// creativecommons.org/licenses/by/4.0/. 
pathogen removal and increase the susceptibility to infections [2]. A high susceptibility to bacterial infections with Neisseria spp. has been observed in complement factor D (CFD) deficient humans [3, 4].

During systemic infections, the complement and coagulation system are tightly networking proteolytic cascades [5]. (1) Platelets and platelet-microparticles (PMP) are able to activate classical and terminal complement activation pathways [6, 7]. (2) Thrombin cleaves C5 through terminal complement activation [8]. (3) FXa, FXIa, and plasmin function as C3 and C5 convertase [9]. In addition, platelet adhesion to injured endothelial cells is promoted by the activated coagulation and complement system [10], i.e., the anaphylatoxin C5a induces tissue factor (TF) activity in human endothelial cells [11]. In turn, activated platelets mediate complement activation via P-selectin (CD62P) expression $[12,13]$. Such interactions may also contribute to thrombosis and thrombocytopenia [14].

One serine protease of the alternative complement activation pathway is the complement factor $\mathrm{D}$ (CFD). CFD is stored in human platelets and is released after stimulation with ADP, collagen or thrombin [15]. The blockade of CFD with an anti-factor D antibody inhibits platelet activation by reduced CD62P expression on the platelet surface [16]. Otherwise, the physiological function of CFD in platelets and its role in coagulation during infection remains unclear. Based on the literature above, we designed a translational study in both mice and men to address the impact of CFD on coagulation in sepsis. We hypothesize (1) that CFD impacts coagulopathy in mice with systemic infection; (2) that CFD interaction with coagulation is associated with outcome in sepsis patients, and (3) that antiplatelet drugs affect the CFD level in patients with sepsis.

\section{Materials and methods}

\section{Study design and interventions of mice experiments}

All animal studies were reviewed and approved by the licensing committee of the state of Lower Saxony (Landesamt für Verbraucherschutz und Lebensmittelsicherheit, Oldenburg, Germany, NRW 550-8594-21-006-089-02), and were in accordance with the German and European guidelines on protection of animals. Our study was designed before the MQTiPSS guidelines for improving animal modeling in sepsis were published [17]. However, we were complying with most of the recommendations regarding study design, human modeling, infection types and organ failure/dysfunction.

Male complement factor D knockout mice $\left(\mathrm{fD}^{-1-}\right)$ were generated as described by $\mathrm{Xu}$ et al. [18], on the background of C57BL/6J. For control studies, male C57BL/6J mice (wild-type) were used. Animals were bred and housed at the animal facility of the Jena University Hospital. For the studies mice were $22 \mathrm{~g}( \pm 3 \mathrm{~g})$ and 12-14 weeks old. Throughout all experiments, animals were kept under standardized conditions with access to food and water ad libitum.

Cecal ligation and puncture (CLP) was performed as described in detail previously [19]. Mice were anesthetized by i.p. injection of ketamine (Ketamin, DeltaSelect, Dreieich, Germany) and xylazine (Rompun, Bayer, Leverkusen, Germany) at a dose of $1 \mu \mathrm{l} / \mathrm{g}$ body weight (BW) (mixture of 10 (ketamine) to 1 (xylazine)). To shortly sum the procedure, after disinfection of the abdominal area the mice were laparotomized by midline incision. Then, $2 / 3$ of the exposed cecum was ligated and punctured through 
and through with a 21-gauge needle [1]. Finally, wound closure was performed by applying simple running sutures to the abdominal musculature and the skin. For the current study, CFD-deficient mice and wild-type mice as comparable control group were undergoing CLP procedure and scored every $3 \mathrm{~h}$, applying the clinical severity score described before [20]. At indicated time points (control, 3, 6 and $24 \mathrm{~h}=4$ groups/genotype) the animals were euthanized and whole blood was drawn. Therefore, anesthetized sham control mice or septic mice were laparotomized and blood was taken by heart puncture.

Blood cell count and sample preparation in mice experiments

Whole blood was taken from septic (six and $24 \mathrm{~h}$ after CLP) and control mice of both genotypes ( $\mathrm{fD}^{-/-}$, wild-type) for full-automatic analysis of leukocytes and platelets counts (PocH-100iV Diff; Sysmex, Leipzig, Germany). The samples for the ROTEM and FACS analyses were used directly after blood drawing. Platelet-rich-plasma (PRP) was prepared by centrifugation at $180 \mathrm{~g}$ for $12 \mathrm{~min}$ at room temperature. Afterwards the PRP was obtain carefully for further analyses. During sample preparation the PRP were kept on $4{ }^{\circ} \mathrm{C}$ to avoid platelets activation. For plasma preparation whole blood was centrifuged at $2000 \times g$ for $10 \mathrm{~min}$. Afterwards, samples were snap-frozen and stored at $-80^{\circ} \mathrm{C}$ for further analysis. For the ELISA tests, plasma samples were thawed on ice before sample preparation.

\section{Rotational thromboelastometry $\left(\right.$ ROTEM $^{\circledR}$, TEM) in mice experiments}

For thromboelastometry (TEM) citrate anticoagulated whole blood from control and septic animals of both genotypes was used (blood volume adapted mixture (10:1) of 9 part of whole blood and 1 part of $3.2 \%$ tri-natrium-citrate solution). Rapidly after sampling whole blood EXTEM (extrinsic coagulation pathway), INTEM (intrinsic coagulation pathway) and FIBTEM (fibrinogen function) test analyses of each group were performed with the ROTEM $^{\circledR}$ delta system (Tem Innovations GmbH, ROTEM ${ }^{\circledR}$ delta system [000200100-DE], EXTEM assay reagents [503-13-20], INTEM assay reagents [503-12-20] and FIBTEM assay reagents [503-16-20] Munich, Germany) according to the manufacturer's instructions.

\section{ELISA of D-dimer and TAT complexes in mice experiments}

D-dimer concentration (Mouse D-dimer ELISA kit [CEA506Mu], Uscn Life Science Inc., Wuhan, China), TAT complexes (Mouse Thrombin-Antithrombin complex ELISA kit [SEA831Mu], Uscn Life Science Inc., Wuhan, China) were measured in septic and control mice plasma of each genotype (wild-type and $\mathrm{fD}^{-/-}$). The minimum of detectable dose of mouse D-dimer was $0.54 \mathrm{ng} / \mathrm{ml}$ and for mouse TAT complexes was $0.004 \mathrm{ng} /$ $\mathrm{ml}$. The ELISAs were performed according to the manufacturer's instructions.

\section{Flow cytometry of GPIIb/Illa and P-selectin surface expression and cytokine levels (CBA assay) in mice experiments}

GPIIb/IIIa and P-selectin surface expression were detected by flow cytometry. Therefore platelet-rich plasma (PRP) was prepared by centrifugation (see above) and stained with antibody for activated form of GPIIb/IIIa (PE Integrin alphaIIbbeta3 [M025-2], $5 \mu \mathrm{l}$ antibody solution to stain $\sim 10^{6}$ platelets in a recommended volume of $25 \mu \mathrm{l}$, recalcification using Tyrode-Hepes buffer containing $1 \mathrm{mM} \mathrm{CaCl} 2$ before flow cytometric analysis, 
Emfret Analytics, Eibelstadt, Germany) and P-selectin (FITC Rat Anti-mouse CD62P [553744], concentration $0.5 \mathrm{mg} / \mathrm{ml}$, Becton Dickinson (BD), East Rutherford, USA) followed by flow cytometric analysis. Plasma cytokine levels were measured using CBA assay (Cytometric Bead Array (CBA) Mouse Inflammation [552364], Becton Dickinson (BD), East Rutherford, USA) by FACS. The plasma samples were collected and analyzed according to the manufacturer's instructions.

\section{Study design and human study population}

The study was conducted in accordance with Helsinki Declaration and approved by the local ethical committee of Jena University Hospital (474403/16). Plasma samples were collected from ICU patients between 2001 and 2005.

Plasma samples were stored at $-80^{\circ} \mathrm{C}$ in the Integrated BioBank Jena (Jena University Hospital, Germany). Informed consent was obtained from all patients or their legal representatives. Inclusion criteria for this analysis were either new onset of sepsis (for the trial patients were evaluated based on the definition of sepsis-1) [21] or presence of systemic inflammation after cardiopulmonary bypass for cardiac surgery. Plasma samples had to be available between 24 and $48 \mathrm{~h}$ after diagnosis of sepsis or SIRS. Patients, who had received fresh frozen plasma or thrombocyte transfusion during their ICU stay, were excluded from this analysis. In total $N=79$ patients were included in the study, whereas the study cohort was divided into two groups: group (1) $\mathrm{N}=45$ infected patients (=diagnosis: sepsis) and group; (2) $\mathrm{N}=35$ non-infected patients (=diagnosis: SIRS). Several parameters were provided from the study group (demographical data, fibrinogen level, C-reactive protein, SOFA [Sepsis-related Organ Failure Assessment] score [22]). The medication history of the study population was evaluated with respect of the use of antiplatelet drugs, especially acetylsalicylic acid (ASA) or clopidogrel. Further, we defined cut-off values of measured complement factor D plasma levels: $<25 \%$ percentiles (CFD levels $\leq 2.4 \mu \mathrm{g} / \mathrm{ml}$ ), $>25 \%$ and $<75 \%$ percentiles (CFD levels $>2.4 \mu \mathrm{g} / \mathrm{ml}$ and $<5.0 \mu \mathrm{g} / \mathrm{ml}$ ), $>75 \%$ percentiles (CFD level $\geq 5.0 \mu \mathrm{g} / \mathrm{ml}$ ).

\section{ELISA of D-dimer, TAT complexes and complement factor D (CFD) in the human study population}

D-dimer (D-Dimer Human Simple Step ELISA ${ }^{\circledR}$ Kit [ab260076], abcam ${ }^{\circledR}$, Cambridge UK), TAT complexes (Human Thrombin-Antithrombin Complex ELISA Kit (TAT) [ab108907], abcam ${ }^{\circledR}$, Cambridge UK) and complement factor D (Quantikine ${ }^{\circledR}$ Human Complement Factor D Immunoassay [DFD00], R\&D Systems ${ }^{\circledR}$, Minneapolis USA) levels were measured in human plasma. The minimum of detectable dose of human D-dimer was $0.07 \mathrm{ng} / \mathrm{ml}$, for human TAT complexes was $1.5 \mathrm{ng} / \mathrm{ml}$, and for human CFD was $0.013 \mathrm{ng} / \mathrm{ml}$. Therefore, ELISAs were performed according to the manufacturer's instructions.

\section{Statistical analyses}

For the animal study, after descriptive statistical analyses, non-parametric tests using Wilcoxon-Mann-Whitney test, or the Kruskal-Wallis test (Dunn's test correction) were performed. For the human study, after descriptive statistical analyses, the obtain data were assessed for normal distribution. For data following normal distribution, we 
applied parametric tests (Student's $t$-test). Otherwise, non-parametric tests (WilcoxonMann-Whitney test or the Kruskal-Wallis test with Dunn's test correction) were used. We report bivariate Pearson correlation coefficients for several variables (CFD, TAT complexes, D-dimer, platelets, SOFA score, modified DIC score). We investigated the association of CFD levels measured after ICU admission and diagnosis sepsis or SIRS for time to in-hospital mortality. The estimated percentiles (25, 50 and 75 percentiles) of the calculated mean CFD plasma level (with confidence interval, CI) of all patients were defined as cut-off limits. Three groups were conducted and the CFD value of each patient of both groups (SIRS and Sepsis) assigned to the groups. Results were displayed in a Kaplan-Meier curve with the classification for CFD levels based on the calculated percentiles (25\%,50\% and 75\%). Using univariate and multivariable Cox regression models, we extended the investigation of CFD level (linear) with time to in-hospital mortality including demographic (sex, age) and clinical variables (TAT complexes, D-dimer, platelets) as well as ICU scores (SOFA, modified DIC score) and state of infection (subgroup sepsis). In multivariate analysis, we adjusted for age and SOFA score which are known confounders when assessing effects on hospital mortality. From these models, we derive hazard ratios (HR) and confidence intervals (CI) with a coverage of $95 \%$. We applied an explorative two-sided significance level of $\alpha=0.05$ and did not correct for multiple testing. Results are depicted as bar plots (mean + SEM) or boxplots (interquartile range [IQR], 0.25, 0.75; whiskers: min, max). Individual data points $(n)$ are plotted over the boxes, or the $\mathrm{n}$ was specified otherwise in the bars or figure legend. The results for patient characteristics are stated as mean with range or min to max. All analyses were done using GraphPad Prism 6 or IBM SPSS 24.

\section{Results}

\section{Coagulation and platelets function in septic $\mathrm{fD}^{-/-}$and wild-type mice}

Leukocyte depletion was found in CFD-deficient $\left(\mathrm{fD}^{-/-}\right)$and wild-type (wt) mice 24-h after CLP (wt: $p=0.0003 ; \mathrm{fD}^{-/-}: p=0.0004$ ) (Fig. 1A). Cytokine levels increased in both groups after sepsis onset (wt: IL-6, $p=0.004$; TNF- $\alpha, p=0.008$; IL-10, $p=0.008 ; \mathrm{fD}^{-1-}$ : IL-6, $p=0.001$; TNF- $\alpha, p=0.002$; IL-10, $p=0.001$ ), whereas IL-6 levels were higher in $\mathrm{fD}^{-1-}$ mice compared to wild-type at 3-h $(p=0.012)$ after CLP (Fig. 1B and Additional file 1: Figure S1D). Platelets counts dropped in wild-type mice $(p=0.003)$ but not in $\mathrm{fD}^{-1-}$ mice 24-h after sepsis induction (Fig. 1A). D-dimer concentrations increased continuously in both groups reaching highest values 24-h after sepsis onset (wt: $p<0.0001$; $\mathrm{fD}^{-1-}: p=0.0006$; Additional file 1: Figure $\mathrm{S1C}$ ). We observed higher values of TAT complexes in control and septic $\mathrm{fD}^{-/-}$mice ( $p=0.02$; Fig. 1D).

All three test settings of rotational thromboelastometry, i.e., EXTEM, INTEM and FIBTEM resulted in enhanced clotting times (ct) in sham control and septic $\mathrm{fD}^{-1-}$ mice. Only the EXTEM ct of sham control $\mathrm{fD}^{-1-}$ mice, which assesses the extrinsic pathway, differed from the wild-type sham control (Fig. 1C, Additional file 1: Figure S1A). CLP was associated with a reduction in maximal clot firmness (mcf) in all three test settings (EXTEM, INTEM and FIBTEM test) in both groups (wt: EXTEM, $p=0.002$; INTEM, $p=0.03$; FIBTEM, $p=0.002$; $\mathrm{fD}^{-1-}$ : EXTEM, $p=0.03$; INTEM, $p=0.004$; FIBTEM, $p=0.009$ ). Further, INTEM and FIBTEM test revealed a lower mcf in septic $\mathrm{fD}^{-1-}$ mice compared to septic wild-type mice (for FIBTEM, Additional file 1: Figure 

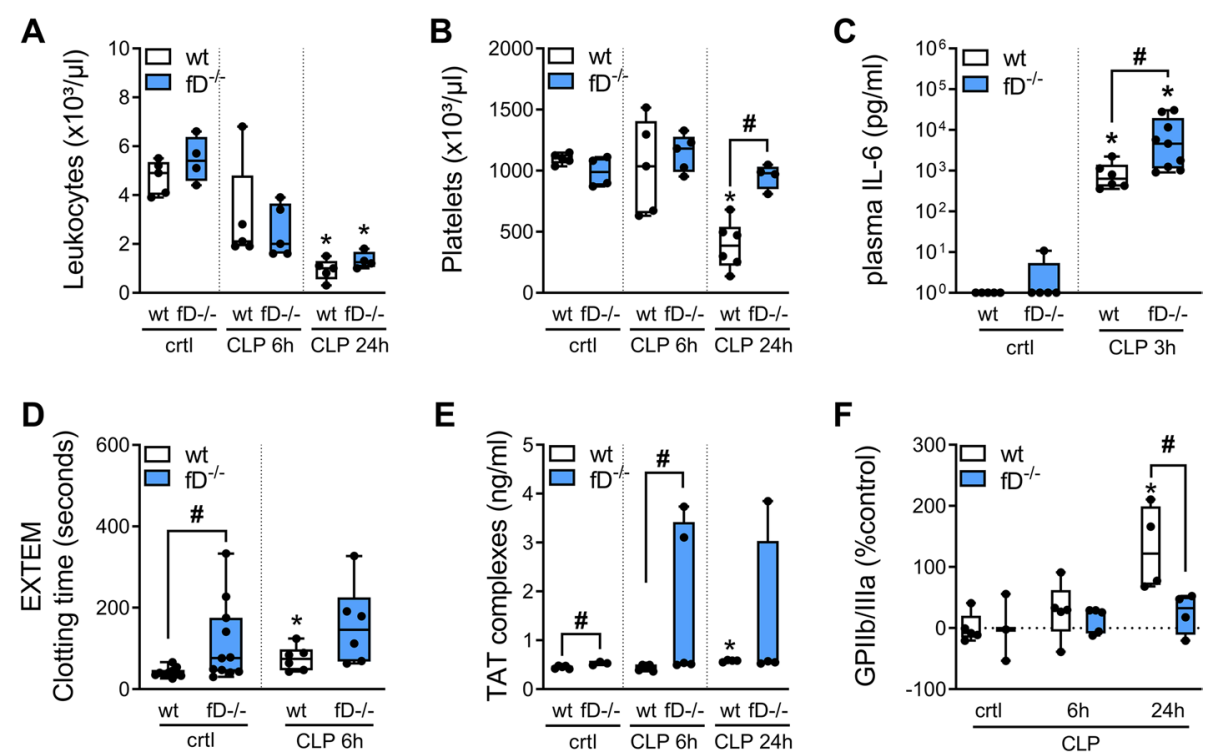

Fig. 1 Complement factor affects immune response and platelets function. A, B Whole blood leukocytes dropped in septic mice (both groups) after CLP. No platelet drop was found in septic $\mathrm{fD}^{-/}$mice $\left(\mathrm{fD}^{-1-}\right.$ ) $24 \mathrm{~h}$ after CLP, whereas platelet counts were reduced in septic wild-type (wt) at same time point. C Plasma cytokine levels of IL-6 were significant higher in septic $\mathrm{fD}^{-/}$mice compared to wild-type three hours after CLP. D EXTEM test were performed and clotting time (ct) in $\mathrm{fD}^{-/-}$mice ( $\left.\mathrm{fD}-/-\right)$ was prolonged in both sham control (crtl) and after CLP. ETAT complexes were found to be higher in control (crtl) and septic $\mathrm{fD}^{-1-}$ mice. F Platelet surface expression of GPIII/IIla was reduced in septic $\mathrm{fD}^{-/-}$mice compared to wild-type $24 \mathrm{~h}$ after CLP. Statistics: ${ }^{*}$ significant to $\mathrm{fD}^{-1-}$ sham control (crtl) or wild-type (wt) sham control (crtl) with $p<0.05,(\mathbf{A}$, $\mathbf{B}, \mathbf{E}$ and $\mathbf{F})$ Kruskal-Wallis test (Dunn's test correction) or (C and D) Wilcoxon-Mann-Whitney test. \#Statistical significance $(p<0.05)$ between $\mathrm{fD}^{-/-}$and wild-type (wt) by means of (A to $\left.\mathbf{F}\right)$ Wilcoxon-Mann-Whitney test. Three to 12 mice/group

S1B). P-selectin expression on isolated platelets increased in both groups after CLP over time but was more pronounced in wild type $24 \mathrm{~h}$ after sepsis induction compared to $\mathrm{fD}^{-1-}$ mice at that time point (wt: $p=0.008 ; \mathrm{fD}^{-1-}: p=0.02$; Additional file 1: Figure S1E). GPIIb/IIIa expression on isolated platelets of septic $\mathrm{fD}^{-/-}$mice did not change relevantly during the experiment, while receptor expression of activated GPIIb/IIIa was enhanced on platelets of wild-type mice 24-h after CLP ( $p=0.03$; Fig. 1E) compared to septic $\mathrm{fD}^{-/-}$mice at that time point and the wild-type control group.

\section{Clinical characteristics in the human study population}

Patient characteristics are summarized in Tables 1, and 2. In short, the sample included a similar number of males and females with a mean age of 66 years (range 29-92 years). Their overall in-hospital mortality was $41 \%$. The most frequent foci were pulmonal (22\%) and abdominal (17\%) infections.

\section{Complement factor D (CFD) levels and their association with antiplatelet drugs} in the human study population

The mean level of complement factor D (CFD) was $4.2 \mu \mathrm{g} / \mathrm{ml}$ (range $0.2-15.0 \mu \mathrm{g} / \mathrm{ml}$, Table 2) at ICU admission. In septic patients the mean CFD value $(4.5 \mu \mathrm{g} / \mathrm{ml}$, Table 2$)$ was higher compared to SIRS patients $(3.8 \mu \mathrm{g} / \mathrm{ml}$, Table 2$)$. 
Table 1 Patient characteristics and pathologies in the investigated study population

\begin{tabular}{|c|c|c|c|c|c|}
\hline \multirow[t]{2}{*}{ Characteristics } & \multicolumn{3}{|l|}{$N$} & \multirow[t]{2}{*}{ Infection } & \multirow[t]{2}{*}{$N$} \\
\hline & Overall & Sepsis & SIRS & & \\
\hline Sex & & & & Sepsis (\%) & $44(56)$ \\
\hline Female & 40 & 22 & 18 & Endocarditis & $4(5)$ \\
\hline Male & 39 & 22 & 17 & Pneumonia & $17(22)$ \\
\hline Age y & & & & Intra-abdominal & $13(16)$ \\
\hline Mean & 66 & 66 & 65 & Pneumonia/intra-abdominal & $4(5)$ \\
\hline Range & $29-92$ & $31-92$ & $29-88$ & Other & $6(8)$ \\
\hline Outcome & & & & SIRS (\%) & $35(44)$ \\
\hline Dead & 32 & 23 & 9 & & \\
\hline Survived & 47 & 21 & 26 & Total & $79(100)$ \\
\hline
\end{tabular}

Table 2 Patient characteristics and scores after diagnosis of sepsis or SIRS in the investigated study population

\begin{tabular}{|c|c|c|c|c|c|c|}
\hline \multirow[t]{2}{*}{ Variables } & \multicolumn{2}{|c|}{ Overall } & \multicolumn{2}{|c|}{ Sepsis } & \multicolumn{2}{|c|}{ SIRS } \\
\hline & $N$ & Mean (min to max) & $N$ & Mean (min to $\max$ ) & $N$ & Mean (min to $\max$ ) \\
\hline In-hospital stay (days) & 78 & $27.2(3-127)$ & 43 & $34.0(6-127)$ & 35 & $18.7(3-70)$ \\
\hline CFD $(\mu \mathrm{g} / \mathrm{ml})$ & 79 & $4.2(0.2-15.0)$ & 44 & $4.5(0.8-15.0)$ & 35 & $3.8(0.2-11.2)$ \\
\hline TAT complexes (ng/ml) & 79 & $18.3(4.7-56.8)$ & 44 & $20.1(5.6-56.8)$ & 35 & $16.0(4.7-43.7)$ \\
\hline D-dimer ( $\mu \mathrm{g} / \mathrm{ml})$ & 79 & $3.4(0.4-29.8)$ & 44 & $4.8(0.6-29.8)$ & 35 & $1.7(0.3-6.3)$ \\
\hline Platelets (Gpt/l) & 79 & $190.7(25-851)$ & 44 & $199.7(25-851)$ & 35 & $179.3(69-515)$ \\
\hline Fibrinogen (g/l) & 73 & $4.5(1.3-10.0)$ & 39 & $5.4(2.1-10)$ & 34 & $3.4(1.3-8.6)$ \\
\hline CRP (mg/l) & 76 & $133.7(4.9-500.0)$ & 44 & $191.1(15-500)$ & 32 & $54.8(4.9-229)$ \\
\hline SOFA score & 78 & $8(1-15)$ & 44 & $9(5-15)$ & 35 & $7(1-14)$ \\
\hline DIC score (modified) & 79 & $3(2-7)$ & 44 & $3(2-7)$ & 35 & $3(2-4)$ \\
\hline
\end{tabular}

The plasma CFD concentration was increased in non-survivors compared to survivors $(5.0 \mu \mathrm{g} / \mathrm{ml}$ in non-survivors to $3.6 \mu \mathrm{g} / \mathrm{ml}$ in survivors, $p=0.015$; Fig. $2 \mathrm{~B})$ at ICU admission. The plasma CFD value of non-survivors and survivors were also compared in both subgroups. Septic non-survivors displayed on average $1.8 \mu \mathrm{g} / \mathrm{ml}$ higher CFD value compared to septic survivors ( $p=0.042$; Additional file 1: Figure S2A). In SIRS patients, CFD value on average differed between survivors and non-survivors by $0.7 \mu \mathrm{g} / \mathrm{ml}$. The CFD concentration of non-survivors without antiplatelet drugs were higher compared to non-survivors with antiplatelet drugs (acetylsalicylic acid [ASA] or clopidogrel; $5.3 \mu \mathrm{g} /$ $\mathrm{ml}$ in patients with no antiplatelet drugs to $4.5 \mu \mathrm{g} / \mathrm{ml}$ in patients with antiplatelet drugs). Non-survivor without antiplatelet drugs displayed significantly higher CFD concentration compared to the survivor with no antiplatelet drugs $(5.3 \mu \mathrm{g} / \mathrm{ml}$ in non-survivors to $3.3 \mu \mathrm{g} / \mathrm{ml}$ in survivors, $p=0.044$; Fig. $2 \mathrm{C}$ ).

\section{Complement factor D (CFD) compared to coagulation parameters and scores of organ dysfunction in the human study population}

D-dimer level and SOFA score were higher in non-survivor compared to survivors in SIRS patients without reaching statistical significance. In sepsis patients, D-dimer level and SOFA score were elevated without being different between non-survivors and 


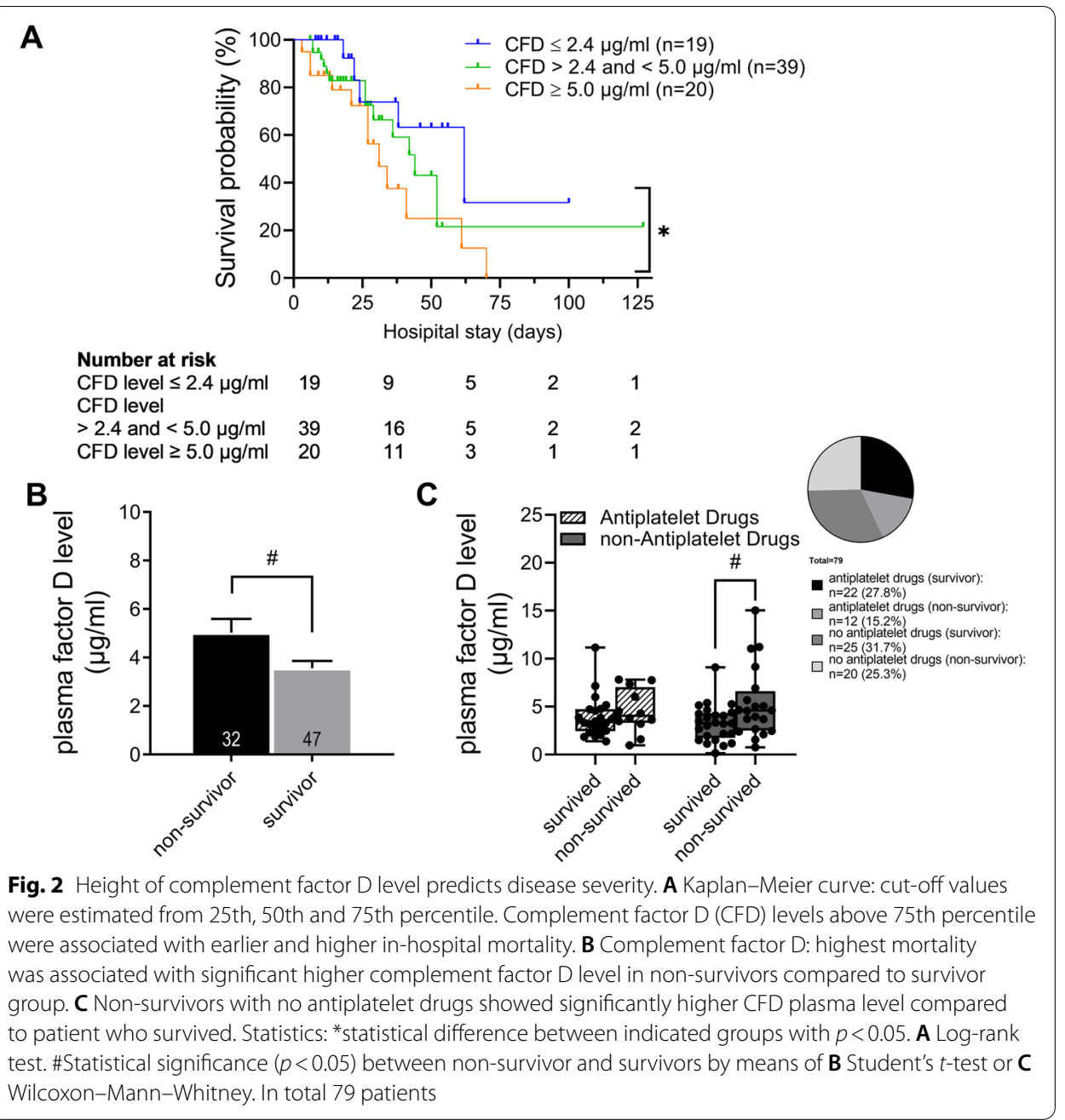

survivors (Additional file 1: Figure S2B, S2C). For TAT complexes and modified DIC score, we observed no evidence for differences between the subgroups (Additional file 1: Figure S2B, S2C). During the first 5 days after ICU admission, platelet counts showed minor differences depending on low $(\leq 2.4 \mu \mathrm{g} / \mathrm{ml})$, mid $(>2.4 \mu \mathrm{g} / \mathrm{ml}$ and $<5.0 \mu \mathrm{g} / \mathrm{ml})$ or high $(\geq 5.0 \mu \mathrm{g} / \mathrm{ml})$ CFD plasma concentration (Additional file 1: Figure S2D). Factor D concentration were positively correlated with the SOFA-score $(r=0.33, p=0.003)$. Platelet counts concentrations were positively correlated with TAT complexes $(r=0.32$, $p=0.004)$ and negatively correlated with both SOFA-score and modified DIC score ( $r=-0.23, p=0.04 ; r=-0.23, p=0.04$; Additional file 1: Table S1, Figure S3).

\section{Complement factor D (CFD) to predict in-hospital mortality in the human study population}

We found higher CFD levels to be associated with an increased in-hospital mortality $(p=0.03$, Table 3 and Fig. 2A).

In the Cox regression analysis, depicted in Table 4, complement factor D (CFD) concentration was independently associated with overall survival when adjusting for age and SOFA score (Table 4). 
Table 3 Association of complement factor D levels to in-hospital mortality

\begin{tabular}{lllll}
\hline CFD level & $N$ (non-survivor) & Percentile & $\begin{array}{l}\text { Median } \\
\text { survival }\end{array}$ & $\mathbf{9 5 \%} \mathbf{C l}$ \\
\hline $\begin{array}{l}\text { Group 1: } \\
\leq 2.4 \mu \mathrm{g} / \mathrm{ml}\end{array}$ & $19(5)$ & 25 th & 62 & $(26.949-97.051)$ \\
$\begin{array}{l}\text { Group 2: } \\
>2.4 \text { and }<5.0 \mu \mathrm{g} / \mathrm{ml}\end{array}$ & $39(14)$ & 50 th & 44 & $(31.494-56.506)$ \\
$\begin{array}{l}\text { Group 3: } \\
\geq 5.0 \mu \mathrm{g} / \mathrm{ml}\end{array}$ & $20(12)$ & 75 th & 31 & $(20.994-41.006)$ \\
Total & & & 42 & $(32.291-51.709)$ \\
\hline
\end{tabular}

Table 4 Regression model results for in-hospital mortality in 78 patients with diagnosis sepsis or SIRS

\begin{tabular}{|c|c|c|c|c|c|c|}
\hline \multirow[t]{3}{*}{ Variables } & \multicolumn{6}{|c|}{ Cox regressions model for in-hospital mortality } \\
\hline & \multicolumn{3}{|c|}{ Univariate models } & \multicolumn{3}{|c|}{ Multivariable models } \\
\hline & Hazard ratio & $95 \% \mathrm{Cl}$ & $p$ value & Hazard ratio & $95 \% \mathrm{Cl}$ & $p$ value \\
\hline CFD & 1.13 & $(1.03-1.25)$ & 0.013 & 1.13 & $(1.00-1.28)$ & 0.043 \\
\hline Age & 1.03 & $(1.00-1.06)$ & 0.049 & 1.02 & $(0.99-1.06)$ & 0.092 \\
\hline Sex & 1.62 & $(0.78-3.36)$ & 0.193 & & & \\
\hline TAT complexes & 0.99 & $(0.96-1.03)$ & 0.679 & & & \\
\hline D-dimer & 1.02 & $(0.93-1.13)$ & 0.636 & & & \\
\hline Platelets & 1.00 & $(0.99-1.00)$ & 0.512 & & & \\
\hline SOFA score & 1.05 & $(0.91-1.21)$ & 0.548 & 0.99 & $(0.84-1.16)$ & 0.869 \\
\hline DIC score (modified) & 1.22 & $(0.80-1.87)$ & 0.360 & & & \\
\hline Sepsis & 0.89 & $(0.40-2.00)$ & 0.784 & & & \\
\hline
\end{tabular}

Statistics: univariate and multivariable Cox regression models (linear) and derived hazard ratios (HR) and confidence intervals $(\mathrm{Cl})$ with a coverage of $95 \%$ of demographic and clinical variables $(p<0.05)$

\section{Discussion}

This translational study supports an important role for the serine protease complement factor D (CFD) in sepsis, both in the mouse as well as in humans: in mice, CFD is associated with a significant impact on coagulation by enhanced platelet function in septic wild-type mice. In humans, high CFD plasma concentration was associated with an increased mortality in sepsis and antiplatelet drugs might favorably outcome affect the CFD plasma level.

While the crosslink between coagulation and complement system is well established $[3-5,7,10-13,23]$, the impact of CFD on the coagulation cascade is still unclear. It is known that CFD is localized in human platelets [15]. Our data add important insights regarding the functional role: an enhanced thrombin generation reflected by higher TAT complex concentration in CFD-deficient mice is not only linked to compensatory C5a formation during sepsis, but also contributes to abnormal coagulation activity. Thrombin is known as a potent platelet activator and initiates GPIIb/IIIa expression on thrombocytes [24]. Interestingly, the surface receptor expression of GPIIb/IIIa was significantly reduced in CFD-deficient mice, while we observed an increase in thrombocyte surface markers in wild-type mice. Other markers of coagulation such as the results of ROTEM (clotting time, maximal clot firmness) or D-dimer were affected 
in CFD-deficient as well as in wild-type mice. So, during infection CFD significantly impacts the inflammation-triggered activation of the coagulation cascade in mice, which was found in wild-type mice by pronounced surface expression of GPIIb/IIIa, P-selectin and the decrease of platelets and hint to the hypercoagulable state during sepsis.

The classical and alternative complement pathways play an essential role to clear endotoxin and to act as critical defense against bacterial infection [25]. Especially in humans, deficiency in factors of the alternative complement pathway such as CFD or properdin is reported to be associated with meningococcal infection and fulminant septic shock $[3,4,26,27]$. In mice, CFD deficiency is associated with the loss of the regulatory role of the alternative pathway. Factor D-deficient mice showed reduced bacterial clearance, increased cytokine levels and fatal outcome [1].

In humans, we found high CFD levels in non-survivors of the whole study population and selectively in sepsis patients. These high CFD levels represent a strong activation of the alternative pathway. Alternative complement activation acts as an amplification loop triggering the hyperactive inflammatory response and thereby contributing negatively to the progression of the disease $[18,28-30]$. Furthermore, CFD is a modulator of complement activation during infection affecting the innate immune response [1]. This might be the reason why a high CFD generation as observed in our study was associated with a high in-hospital mortality. Further, the positive correlation between SOFA score and CFD would suggest a negative effect regarding (multi-)organ dysfunction in patients with strongly elevated CFD plasma level. An important aspect to monitor disease severity is to identify the individual patient risk based on diagnosis, co-morbidity and previous surgical procedures in the light of activation of coagulation and immune responses. An increased C3 and C4 consumption in sepsis, found by inversely lower C3 and C4 levels in patient plasma, is more often associated with unfavorable outcome [31]. Complement activation as an indicator of hospital acquired infection has a strong impact on mortality and hospital stay. Also, the depletion of complement C3 was found to be connected to poor prognosis in severe abdominal sepsis [32].

Our data indicate that the alternative complement pathway interacts with immune processes and coagulation during infection and inflammation. Factor D seems to play a crucial role in this interaction during sepsis. Other authors focused on terminal complement activation and the lectin pathway. High C3, MAC, and MBL serum concentrations are predictive for sepsis-induced disseminated intravascular coagulation [33].

In sepsis, thrombomodulin is a strong predictor of (multi-)organ dysfunction [34]. Hemostasis-related parameters like aPTT, PT and D-dimer are associated with severity of the sepsis [35]. Further, endogenous thrombin can distinguish between beneficial and hazardous hemostatic alterations [36]. Based on the knowledge of dissimilarities in coagulation abnormalities in mice and human, we could not confirm the results of other authors, which found higher D-dimer and TAT complexes associated with disease severity in non-survivors [37].

Based on our findings in the animal experiments regarding dysregulated platelet function, we evaluated the effects of a medication with antiplatelets drugs in our study population. Our data showed reduced CFD plasma level in patients on antiplatelet drugs compared to patients without this medication. So, these could be responsible to improve 
the septic hypercoagulable state in the patient cohort. These results are in line with the observation in CFD-deficient mice, which shows reduced surface receptor expression on thrombocytes and no relevant platelet drops after sepsis induction over time. Previous studies reported the prevention of organ dysfunction by antiplatelet drugs [38, 39] and low-dose aspirin as a therapeutic option to prevent organ failure [40]. It is hypothesized, that antiplatelet drugs (ASA, clopidogrel) attenuates the expression and release of immune markers (complement factors such as $\mathrm{C} 4 \mathrm{~b}$ ), inflammatory cytokines (including pro- and anti-inflammatory effectors, e.g., TNF- $\alpha$, IL-1, -6, -10, -13), and induces transcription factor $\mathrm{NF}_{\mathrm{K} B}$ during inflammation and cellular stress response [41].

\section{Limitations}

The following limitations have to be acknowledged for the present study. (1) The clinical study was designed as retrospective data analysis in a small and selective study group with all its advantages and disadvantages. (2) Patients were recruited from a surgical ICU. Surgical procedures on its own may affect the predictive role of CFD and its impact to in-hospital mortality. More studies are necessary to validate the clinical impact of CFD as potential biomarker and potential discriminator of in-hospital outcome.

\section{Conclusions}

The crosslink between complement and coagulation cascades seems to be permanently present in the course of systemic inflammation. In mice, CFD is linked to pronounced platelet activation, depicted by higher surface expression on thrombocytes in sepsis. In humans, CFD revealed significant impact on in-hospital mortality and predicts disease severity and poor outcome shown by high factor D level in the non-survivor group. The impact of antiplatelet drugs to improve sepsis might be beneficial and affect CFD plasma level and patient outcome.

\footnotetext{
Abbreviations

ADP: Adenosine diphosphate; ASA: Acetylsalicylic acid; aPTT: Activated partial thromboplastin time; BW: Body weight; CBA: Cytometric bead array; CD62P: P-selectin; CFD: Complement factor D; Cl: Confidence interval; CLP: Cecal ligation and puncture; CRP: C-reactive protein; Ct: Clotting time; C3,-4, -5, -5a: Complement factor 3, -4, -5, -5a; DIC: Disseminated intravascular coagulation; EXTEM: Extrinsic coagulation pathway; FACS: Fluorescence-activated cell sorting; $\mathrm{fD}^{-/-}$: Complement factor D knock out mice; FIBTEM: Fibrinogen function; FITC: Fluorescein-5-isothiocyanate; FXa, -Xia: Factor Xa, -Xla; GPIIb/IIla: Glycoprotein IIb/IIla; HR: Hazard ratio; ICU: Intensive care unit; IL-6: Interleukin-6; IL-10: Interleukin-10; INTEM: Intrinsic coagulation pathway; Mcf: Maximal clot firmness; MAC: Membrane attack complex; MBL: Mannosebinding lectin; NFkB: Nuclear factor-kappa B; PE: Phycoerythrin; PMP: Platelet-microparticles; PRP: Platelet-rich-plasma; PT: Prothrombin time; ROTEM: Rotational thromboelastometry; SIRS: Systemic inflammatory response syndrome; SOFA: Sepsis-related Organ Failure Assessment; TAT complex: Thrombin-antithrombin complex; TNFa: Tumor necrosis factor-a; TF: Tissue factor; Wt: Wild-type mice.
}

\section{Supplementary Information}

The online version contains supplementary material available at https://doi.org/10.1186/s40635-021-00405-8.

Additional file 1. Complement factor $\mathrm{D}$ is linked to platelet activation in human and rodent sepsis.

\section{Acknowledgements}

The authors acknowledge Ulrike Vetterling for supporting animal experiments and Edith Walther for excellent technical assistance. The animal studies were further supported by a grant of Deutsche Forschungsgemeinschaft (RI 1216/4-1). Collection and storage of the biomaterials were supported by a grant from the Bundesministerium für Bildung und Forschung (0312617) and by the Interdisziplinäres Zentrum für Klinische Forschung Jena (TP 4.3). Michael Kiehntopf and Cornelius Eibner were responsible for and facilitated storage of all biomaterials in a standardized fashion in the IBBJ (Integrated Biomaterial Bank Jena, Jena University Hospital, Germany) 


\section{Authors' contributions}

We ensure that all authors had seen and approved the final version of this manuscript and contributed to it as stated here: In vivo experiments and data settings contributing mice experiments was performed and analysed by KD, MS and OS. FB and OS designed and performed human study trial. AS guided statistical analysis. FB, MB and RAC supervised and guided the study. All authors wrote the manuscript. All authors read and approved the final manuscript.

\section{Funding}

Open Access funding enabled and organized by Projekt DEAL. This work was funded by Deutsche Forschungsgemeinschaft (RI 1216/4-1) and supported by Bundesministerium für Bildung und Forschung (0312617) and the Interdisziplinäres Zentrum für Klinische Forschung Jena (TP 4.3).

\section{Availability of data and materials}

The datasets used and/or analysed during the current study are available from the corresponding author on reasonable request.

\section{Declarations}

\section{Ethical approval and consent to participate}

All animal studies: reviewed and approved by the licensing committee of the state of Lower Saxony (Landesamt für Verbraucherschutz und Lebensmittelsicherheit, Oldenburg, Germany, NRW 550-8594-21-006-089-02), and in accordance with the German and European guidelines on protection of animals. The human study: conducted in accordance with Helsinki Declaration and approved by the local ethical committee of Jena University Hospital (474403/16). Informed consent: obtained from all patients or their legal representatives.

\section{Consent for publication}

Not applicable.

Competing interests

The author(s) declare no competing interests.

\section{Author details}

${ }^{1}$ Department of Anesthesiology and Intensive Care Medicine, Jena University Hospital, Jena, Germany. ${ }^{2}$ Center for Sepsis Control and Care (CSCC), Jena University Hospital, Jena, Germany. ${ }^{3}$ Institute of Medical Statistics, Computer and Data Sciences, Jena University Hospital, Jena, Germany.

Received: 10 March 2021 Accepted: 20 July 2021

Published online: 16 August 2021

\section{References}

1. Dahlke K, Wrann CD, Sommerfeld O et al (2011) distinct different contributions of the alternative and classical complement activation pathway for the innate host response during sepsis. J Immunol 186:3066-3075. https://doi.org/10. 4049/jimmunol.1002741

2. Mayilyan KR (2012) Complement genetics, deficiencies, and disease associations. Protein Cell 3:487-496. https://doi.org/ 10.1007/s13238-012-2924-6

3. Hiemstra PS, Langeler E, Compier B et al (1989) Complete and partial deficiencies of complement factor D in a Dutch family. J Clin Invest 84:1957-1961. https://doi.org/10.1172/JCl1 14384

4. Sprong T, Roos D, Weemaes C et al (2006) Deficient alternative complement pathway activation due to factor D deficiency by 2 novel mutations in the complement factor $\mathrm{D}$ gene in a family with meningococcal infections. Blood 107:4865-4870. https://doi.org/10.1182/blood-2005-07-2820

5. Markiewski MM, Nilsson B, Nilsson Ekdahl Ket al (2007) Complement and coagulation: strangers or partners in crime? Trends Immunol 28:184-192. https://doi.org/10.1016/j.it.2007.02.006

6. Peerschke EIB, Yin W, Grigg SE, Ghebrehiwet B (2006) Blood platelets activate the classical pathway of human complement. JThromb Haemost 4:2035-2042. https://doi.org/10.1111/j.1538-7836.2006.02065.x

7. Davis AE (2004) Biological effects of C1 inhibitor. Drug News Perspect 17:439-446

8. Huber-Lang M, Sarma JV, Zetoune FS et al (2006) Generation of C5a in the absence of C3: a new complement activation pathway. Nat Med 12:682-687. https://doi.org/10.1038/nm1419

9. Amara U, Rittirsch D, Flierl M et al (2008) Interaction between the coagulation and complement system. Adv Exp Med Biol 632:71-79

10. Rayes J, Roumenina LT, Dimitrov JD et al (2014) The interaction between factor $\mathrm{H}$ and WWF increases factor $\mathrm{H}$ cofactor activity and regulates VWF prothrombotic status. Blood 123:121-125. https://doi.org/10.1182/blood-2013-04-495853

11. Ikeda K, Nagasawa K, Horiuchi T et al (1997) C5a induces tissue factor activity on endothelial cells. Thromb Haemost 77:394-398

12. del Conde I, Crúz MA, Zhang H et al (2005) Platelet activation leads to activation and propagation of the complement system. J Exp Med 201:871-879. https://doi.org/10.1084/jem.20041497

13. Saggu G, Cortes C, Emch HN et al (2013) Identification of a novel mode of complement activation on stimulated platelets mediated by properdin and C3(H2O). J Immunol 190:6457-6467. https://doi.org/10.4049/jimmunol.1300610

14. Peerschke EI, Yin W, Ghebrehiwet B (2010) Complement activation on platelets: Implications for vascular inflammation and thrombosis. Mol Immunol 47:2170-2175. https://doi.org/10.1016/j.molimm.2010.05.009 
15. Kenney DM, Davis AE (1981) Association of alternative complement pathway components with human blood platelets: secretion and localization of factor D and beta 1H Globulin. Clin Immunol Immunopathol 21:351-363

16. Fung M, Loubser PG, Ündar A et al (2001) Inhibition of complement, neutrophil, and platelet activation by an anti-factor D monoclonal antibody in simulated cardiopulmonary bypass circuits. J Thorac Cardiovasc Surg 122:113-122. https:// doi.org/10.1067/mtc.2001.114777

17. Osuchowski MF, Ayala A, Bahrami S et al (2018) Minimum quality threshold in pre-clinical sepsis studies (MQTiPSS): an international expert consensus initiative for improvement of animal modeling in sepsis. Intensive Care Med Exp 6:26. https://doi.org/10.1186/s40635-018-0189-y

18. XuY, Ma M, Ippolito GC et al (2001) Complement activation in factor D-deficient mice. Proc Natl Acad Sci 98:1457714582. https://doi.org/10.1073/pnas.261428398

19. Rittirsch D, Huber-Lang MS, Flierl MA, Ward PA (2009) Immunodesign of experimental sepsis by cecal ligation and puncture. Nat Protoc 4:31-36. https://doi.org/10.1038/nprot.2008.214

20. Gonnert FA, Recknagel P, Seidel M et al (2011) Characteristics of clinical sepsis reflected in a reliable and reproducible rodent sepsis model. J Surg Res 170:e123-134. https://doi.org/10.1016/j.jss.2011.05.019

21. Bone RC, Balk RA, Cerra FB et al (1992) Definitions for sepsis and organ failure and guidelines for the use of innovative therapies in sepsis. The ACCP/SCCM Consensus Conference Committee. American College of Chest Physicians/Society of Critical Care Medicine. Chest 101:1644-1655. https://doi.org/10.1378/chest.101.6.1644

22. Vincent J-L, Moreno R, Takala J et al (1996) The SOFA (Sepsis-related Organ Failure Assessment) score to describe organ dysfunction/failure: on behalf of the Working Group on Sepsis-Related Problems of the European Society of Intensive Care Medicine (see contributors to the project in the appendix). Intensive Care Med 22:707-710. https://doi.org/10. 1007/BF01709751

23. Yin W, Ghebrehiwet B, Peerschke EIB (2008) Expression of complement components and inhibitors on platelet microparticles. Platelets 19:225-233. https://doi.org/10.1080/09537100701777311

24. Kahn ML, Zheng Y-W, Huang W et al (1998) A dual thrombin receptor system for platelet activation. Nature 394:690-694. https://doi.org/10.1038/29325

25. Charchaflieh J, Wei J, Labaze G et al (2012) The role of complement system in septic shock. Clin Dev Immunol 2012:1-8. https://doi.org/10.1155/2012/407324

26. Derkx HH, Kuijper EJ, Fijen CA et al (1995) Inherited complement deficiency in children surviving fulminant meningococcal septic shock. Eur J Pediatr 154:735-738

27. Genel F, Atlihan F, Gulez N et al (2006) Properdin deficiency in a boy with fulminant meningococcal septic shock. Acta Paediatr 95:1498-1500. https://doi.org/10.1080/08035250600603008

28. Taube C, Thurman JM, Takeda K et al (2006) Factor B of the alternative complement pathway regulates development of airway hyperresponsiveness and inflammation. Proc Natl Acad Sci USA 103:8084-8089. https://doi.org/10.1073/pnas. 0602357103

29. Thurman JM, Holers VM (2006) The central role of the alternative complement pathway in human disease. J Immunol (Baltimore Md: 1950) 176:1305-1310. https://doi.org/10.4049/jimmunol.176.3.1305

30. Turnberg D, Lewis M, Moss J et al (2006) Complement activation contributes to both glomerular and tubulointerstitial damage in adriamycin nephropathy in mice. J Immunol (Baltimore Md: 1950) 177:4094-4102. https://doi.org/10.4049/ jimmunol.177.6.4094

31. Lendak D, Mihajlovic D, Mitic G et al (2018) Complement component consumption in sepsis correlates better with hemostatic system parameters than with inflammatory biomarkers. Thromb Res 170:126-132. https://doi.org/10.1016/j. thromres.2018.08.013

32. Ren J, Zhao Y, Yuan Y et al (2012) Complement depletion deteriorates clinical outcomes of severe abdominal sepsis: a conspirator of infection and coagulopathy in crime? PLOS ONE 7:e47095. https://doi.org/10.1371/journal.pone.0047095

33. Zhao X, Chen Y-X, Li C-S (2015) Predictive value of the complement system for sepsis-induced disseminated intravascular coagulation in septic patients in emergency department. J Crit Care 30:290-295. https://doi.org/10.1016/j.jcrc.2014. 11.007

34. Mihajlovic DM, Lendak DF, Draskovic BG et al (2015) Thrombomodulin is a strong predictor of multiorgan dysfunction syndrome in patients with sepsis. Clin Appl Thromb Hemost 21:469-474. https://doi.org/10.1177/1076029613508600

35. Mihajlovic D, Lendak D, Mitic G et al (2015) Prognostic value of hemostasis-related parameters for prediction of organ dysfunction and mortality in sepsis. Turk J Med Sci 45:93-98

36. Mihajlovic D, Brkic S, Lendak D et al (2017) Endogenous thrombin potential as marker of procoagulant response that can be useful in early stage of sepsis. Blood Coagul Fibrinolysis 28:460-467. https://doi.org/10.1097/MBC.0000000000 000622

37. Kinasewitz GT, Yan SB, Basson B et al (2004) Universal changes in biomarkers of coagulation and inflammation occur in patients with severe sepsis, regardless of causative micro-organism [ISRCTN74215569]. Crit Care 8:R82-90. https://doi. org/10.1186/cc2459

38. Winning J, Neumann J, Kohl M et al (2010) Antiplatelet drugs and outcome in mixed admissions to an intensive care unit. Crit Care Med 38:32-37. https://doi.org/10.1097/CCM.0b013e3181b4275c

39. Otto GP, Sossdorf M, Boettel J et al (2013) Effects of low-dose acetylsalicylic acid and atherosclerotic vascular diseases on the outcome in patients with severe sepsis or septic shock. Platelets 24:480-485. https://doi.org/10.3109/09537104. 2012.724482

40. Lösche W, Boettel J, Kabisch B et al (2012) Do aspirin and other antiplatelet drugs reduce the mortality in critically III patients? Thrombosis 2012:1-8. https://doi.org/10.1155/2012/720254

41. Akinosoglou K, Alexopoulos D (2014) Use of antiplatelet agents in sepsis: a glimpse into the future. Thromb Res 133:131-138. https://doi.org/10.1016/j.thromres.2013.07.002

\section{Publisher's Note}

Springer Nature remains neutral with regard to jurisdictional claims in published maps and institutional affiliations. 